\title{
A Generalization of the Hyers -Ulam-Aoki Type Stability of Some Banach Lattice-Valued Functional Equation
}

\author{
Nutefe Kwami Agbeko, Patrícia Szokol \\ Institute of Mathematics, University of Miskolc, 3515 Miskolc-Egyetemváros, Hungary \\ matagbek@uni-miskolc.hu \\ Institute of Mathematics, MTA-DE Research Group "Equations, Functions and Curves", \\ Hungarian Academy of Sciences and University of Debrecen, \\ P. O. Box 12, 4010 Debrecen, Hungary \\ szokol.patricia@inf.unideb.hu
}

Presented by Pier L. Papini

Received April 30, 2017

Abstract: We obtained a generalization of the stability of some Banach lattice-valued functional equation with the addition replaced in the Cauchy functional equation by lattice operations and their combinations.

Key words: Banach lattices, Hyers-Ulam-Aoki type of stability.

AMS Subject Class. (2010): 39B82, 46B30, 46B42.

\section{INTRODUCTION}

All along $\left(\mathcal{X}, \wedge_{\mathcal{X}}, \vee_{\mathcal{X}}\right)$ will stand for a normed Riesz space and $\left(\mathcal{Y}, \wedge_{\mathcal{Y}}, \vee_{\mathcal{Y}}\right)$ for a Banach lattice with $\mathcal{X}^{+}$and $\mathcal{Y}^{+}$their respective positive cones. Let us pose the following problem.

Problem 1. Given three numbers $\varepsilon, p, q \in(0, \infty)$, two Riesz spaces $G_{1}$ and $G_{2}$ with $G_{2}$ being endowed with a metric $d(\cdot, \cdot)$, four lattice operations $\Delta_{G_{1}}^{*}, \Delta_{G_{1}}^{* *} \in\left\{\wedge_{G_{1}}, \vee_{G_{1}}\right\}$ and $\Delta_{G_{2}}^{*}, \Delta_{G_{2}}^{* *} \in\left\{\wedge_{G_{2}}, \vee_{G_{2}}\right\}$, does there exist some real number $\delta>0$ such that, if a mapping $F: G_{1} \rightarrow G_{2}$ satisfies

$$
\begin{aligned}
d\left(\left(F\left(\left(\tau^{q}|x t|\right) \Delta_{G_{1}}^{*}\left(\eta^{q}|y|\right)\right)\right) \Delta_{G_{2}}^{*}\left(F\left(\left(\tau^{q}|x|\right) \Delta_{G_{1}}^{* *}\left(\eta^{q}|y|\right)\right)\right),\right. & \\
\left.\left(\tau^{p} F(|x|)\right) \Delta_{G_{2}}^{* *}\left(\eta^{p} F(|y|)\right)\right) & \leq \delta
\end{aligned}
$$

for all $x, y \in G_{1}$ and all $\tau, \eta \in[0, \infty)$, then an operation-preserving functional $T: G_{1} \rightarrow G_{2}$ exists with the property that

$$
d(T(x), F(x)) \leq \varepsilon
$$


for all $x \in G_{1}$ and all $\tau, \eta \in[0, \infty)$ ?

If in (1.1) we let $\tau=\eta=1$, then the above problem reduces to the problem posed and treated in [5].

The study of functional equations and inequalities in lattice environments is motivated by the fact that many addition-related results or theorems can be extended and can be proved mutatis mutandis. For more references about the earliest extensions of the kind, we would refer the reader to the papers $[1,2,3,4]$.

The main goal of this paper is to show how Ulam-Hyers-Aoki styled version of perturbation (1.1) leads to the unique solution of the functional equation

$$
\begin{aligned}
\left(T\left(\left(\tau^{q}|x|\right) \Delta_{\mathcal{X}}^{*}\left(\eta^{q}|y|\right)\right)\right) \Delta_{\mathcal{Y}}^{*} & \left(T\left(\left(\tau^{q}|x|\right) \Delta_{\mathcal{X}}^{* *}\left(\eta^{q}|y|\right)\right)\right) \\
= & \left(\tau^{p} T(|x|)\right) \Delta_{\mathcal{Y}}^{* *}\left(\eta^{p} T(|y|)\right)
\end{aligned}
$$

for all $x, y \in \mathcal{X}$ and all $\tau, \eta \in[0, \infty)$, where $\Delta_{\mathcal{X}}^{*}, \Delta_{\mathcal{X}}^{* *} \in\left\{\wedge_{\mathcal{X}}, \vee_{\mathcal{X}}\right\}$ and $\Delta_{\mathcal{Y}}^{*}, \Delta_{\mathcal{Y}}^{* *} \in\left\{\wedge \mathcal{Y}, \vee_{\mathcal{Y}}\right\}$ are fixed lattice operations.

Remark 1.1. If we let $\eta=\tau$ and $y=x$ in equation (1.2), then we observe that

$$
T\left(\tau^{q}|x|\right)=\tau^{p} T(|x|)
$$

for all $x \in \mathcal{X}$ and all $\tau \in[0, \infty)$.

The results we obtained are straightforward generalizations of Agbeko [3, $5,6]$ and Salahi et al [16]. For an additional reference we would like to mention the paper [7] where we proved separation and stability results for operators mapping a semi-group with values in a Riesz lattice.

We recall that a functional $H: \mathcal{X} \rightarrow \mathcal{Y}$ is cone-related if $H\left(\mathcal{X}^{+}\right)=$ $\{H(|x|): x \in \mathcal{X}\} \subset \mathcal{Y}^{+}$(see more about this notion in $[3,4]$ ).

Some few references about Hyers-Ulam stability problems and solutions can be found, e.g. in $[8,11,13,14,15]$.

Our theorems will be deduced from the following Forti's result [10].

Theorem 1.1. (Forti) Let $(X, d)$ be a complete metric space and $S$ an appropriate set. Assume some functions $f: S \rightarrow X, G: S \rightarrow S, H: X \rightarrow X$ and $\delta: S \rightarrow[0, \infty)$ satisfy the inequality

$$
d(H(f(G(x))), f(x)) \leq \delta(x)
$$


for all $x \in S$. If function $H$ both is continuous and satisfies the inequality

$$
d(H(u), H(v)) \leq \varphi(d(u, v)), \quad u, v \in X
$$

for a certain non-decreasing subadditive function $\varphi:[0, \infty) \rightarrow[0, \infty)$ and the series

$$
\sum_{j=0}^{\infty} \varphi^{j}\left(\delta\left(G^{j}(x)\right)\right)
$$

is convergent for every $x \in S$, then there exists a unique function $F: S \rightarrow X$ solution of the functional equation

$$
H(F(G(x)))=F(x), \quad x \in S,
$$

and satisfying the following inequality:

$$
d(F(x), f(x)) \leq \sum_{j=0}^{\infty} \varphi^{j}\left(\delta\left(G^{j}(x)\right)\right) .
$$

The function $F$ is given by

$$
F(x)=\lim _{n \rightarrow \infty} H^{n}\left(f\left(G^{n}(x)\right)\right) .
$$

\section{The MAIN RESUlts}

Theorem 2.1. Given a pair of real numbers $(p, q) \in(0, \infty) \times(0, \infty)$, consider a cone-related functional $F: \mathcal{X} \rightarrow \mathcal{Y}$ for which there are numbers $\vartheta>0$ and $\alpha$ with $q \alpha \in(p, \infty)$ such that

$$
\begin{aligned}
\| F\left(\left(\tau^{q}|x|\right) \Delta_{\mathcal{X}}^{*}\left(\eta^{q}|y|\right)\right) \Delta_{\mathcal{Y}}^{*} F\left(\left(\tau^{q}|x|\right) \Delta_{\mathcal{X}}^{* *}\left(\eta^{q}|y|\right)\right) \\
-\left(\tau^{p} F(|x|)\right) \Delta_{\mathcal{Y}}^{* *}\left(\eta^{p} F(|y|)\right) \| \leq 2^{(p-1)} \vartheta\left(\|x\|^{\alpha}+\|y\|^{\alpha}\right)
\end{aligned}
$$

for all $x, y \in \mathcal{X}$ and all $\tau, \eta \in[0, \infty)$. Then the sequence $\left(2^{n p} F\left(2^{-n q}|x|\right)\right)_{n \in \mathbb{N}}$ is a Cauchy sequence for every $x \in \mathcal{X}$. Let the functional $T: \mathcal{X}^{+} \rightarrow \mathcal{Y}^{+}$be defined by

$$
T(|x|)=\lim _{n \rightarrow \infty} 2^{n p} F\left(2^{-n q}|x|\right)
$$

for all $x \in \mathcal{X}$. Then $T$ both is the unique solution of (1.2) and satisfies inequality

$$
\|T(|x|)-F(|x|)\| \leq \frac{2^{p} \vartheta}{2^{q \alpha}-2^{p}}\|x\|^{\alpha}
$$

for every $x \in \mathcal{X}$. 
Theorem 2.2. Given a pair of real numbers $(p, q) \in(0, \infty) \times(0, \infty)$, consider a cone-related functional $F: \mathcal{X} \rightarrow \mathcal{Y}$ for which there are numbers $\beta \in[0, \infty), \vartheta>0$ and $\alpha$ with $q \alpha \in(0, p)$ such that

$$
\begin{aligned}
\| F\left(\left(\tau^{q}|x|\right) \Delta_{\mathcal{X}}^{*}\left(\eta^{q}|y|\right)\right) \Delta_{\mathcal{Y}}^{*} F\left(\left(\tau^{q}|x|\right) \Delta_{\mathcal{X}}^{* *}\left(\eta^{q}|y|\right)\right) \\
-\left(\tau^{p} F(|x|)\right) \Delta_{\mathcal{Y}}^{* *}\left(\eta^{p} F(|y|)\right) \| \leq \beta+\vartheta 2^{-(p+1)}\left(\|x\|^{\alpha}+\|y\|^{\alpha}\right)
\end{aligned}
$$

for all $x, y \in \mathcal{X}$ and all $\tau, \eta \in[0, \infty)$. Then the sequence $\left(2^{-n p} F\left(2^{n q}|x|\right)\right)_{n \in \mathbb{N}}$ is a Cauchy sequence for every fixed $x \in \mathcal{X}$. Let the functional $T: \mathcal{X}^{+} \rightarrow \mathcal{Y}^{+}$ be defined by

$$
T(|x|)=\lim _{n \rightarrow \infty} 2^{-n p} F\left(2^{n q}|x|\right)
$$

for all $x \in \mathcal{X}$. Then $T$ both is the unique solution of (1.2) and satisfies inequality

$$
\|T(|x|)-F(|x|)\| \leq \frac{\beta 2^{p}}{2^{p}-1}+\frac{\vartheta\|x\|^{\alpha} 2^{q \alpha}}{2^{p}-2^{q \alpha}}
$$

for every $x \in \mathcal{X}$.

Remark 2.1. If the conditions of Theorem 2.1 or Theorem 2.2 hold true, then $F(0)=0$.

Proof. The proof is similar to its counterpart in $[5,6]$ under the conditions of Theorem 2.1 or Theorem 2.2 when $\beta=0$. Under the condition of Theorem 2.2 with $\beta>0$, we need to prove that $F(0)=0$. Suppose in the contrary that $F(0)>0$ were true. Then by letting $x=y=0$ and $\eta=\tau$ in (2.4), inequality

$$
\left\|F(0)-\tau^{p} F(0)\right\| \leq \beta
$$

follows or, equivalently

$$
\left|\tau^{p}-1\right| \leq \frac{\beta}{\|F(0)\|}<\infty,
$$

which, as $\tau$ tends to infinity, would lead to an absurdity, indeed. Hence the relation $F(0)=0$ must be true.

Remark 2.2. Let $Z$ be a set closed under the scalar multiplication, i.e. $b z \in Z$ whenever $b \in \mathbb{R}$ and $z \in Z$. Given a number $c \in \mathbb{R}$ let the function $\gamma: Z \rightarrow Z$ be defined by $\gamma(z)=c z$. Then $\gamma^{j}: Z \rightarrow Z$ the $j$-th iteration of $\gamma$ is given by $\gamma^{j}(z)=c^{j} z$ for every natural number $j \geq 2$. 


\section{Proof of the main Results}

We shall use the technique in [5] to prove the main theorems.

Proof of Theorem 2.1. First, if we choose $\tau=\eta=2, y=x$ and replace $x$ by $2^{-q} x$ in inequality (2.1) then we obviously have

$$
\left\|2^{p} F\left(2^{-q}|x|\right)-F(|x|)\right\| \leq \vartheta 2^{p-q \alpha}\|x\|^{\alpha} .
$$

Next, let us define the following functions:

$$
\begin{array}{lll}
G: \mathcal{X}^{+} \rightarrow \mathcal{X}^{+}, & G(|x|)=2^{-q}|x|, & \text { for all } x \in \mathcal{X}, \\
\delta: \mathcal{X}^{+} \rightarrow[0, \infty), & \delta(|x|)=\vartheta 2^{p-q \alpha}\|x\|^{\alpha}, & \text { for all } x \in \mathcal{X}, \\
\varphi:[0, \infty) \rightarrow[0, \infty), & \varphi(t)=2^{p} t, & \\
H: \mathcal{Y}^{+} \rightarrow \mathcal{Y}^{+}, & H(|y|)=2^{p}|y|, & \text { for all } y \in \mathcal{Y}, \\
d(\cdot, \cdot): \mathcal{Y}^{+} \times \mathcal{Y}^{+} \rightarrow[0, \infty), & d\left(\left|y_{1}\right|,\left|y_{2}\right|\right)=\left\|\left|y_{1}\right|-\left|y_{2}\right|\right\|, & \text { for all } y_{1}, y_{2} \in \mathcal{Y} .
\end{array}
$$

We shall verify the fulfilment of all the three conditions of the Forti's theorem as follows.

(I) From inequality (3.1) we obviously have

$$
\begin{aligned}
d(H(F(G(|x t|))), F(|x|)) & =\|H(F(G(|x|)))-F(|x|)\| \\
& =\left\|2^{p} F\left(2^{-q}|x|\right)-F(|x|)\right\| \\
& \leq \vartheta 2^{p-q \alpha}\|x\|^{\alpha}=\delta(|x|) .
\end{aligned}
$$

(II) $d\left(H\left(\left|y_{1}\right|\right), H\left(\left|y_{2}\right|\right)\right)=2^{p} \|\left|y_{1}\right|-\left|y_{2}\right|||=\varphi\left(d\left(\left|y_{1}\right|,\left|y_{2}\right|\right)\right)$ for all $y_{1}, y_{2} \in \mathcal{Y}$.

(III) Clearly, on the one hand $\varphi$ is a non-decreasing subadditive function on the positive half line, and on the other hand by applying Remark 2.2 on both the iterations $G^{j}$ and $\varphi^{j}$ of $G$ and $\varphi$ respectively, one can observe that

$$
\sum_{j=0}^{\infty} \varphi^{j}\left(\delta\left(G^{j}(|x|)\right)\right)=\vartheta 2^{p-q \alpha}\|x\|^{\alpha} \sum_{j=0}^{\infty} 2^{(p-q \alpha) j}=\vartheta\|x\|^{\alpha} \frac{2^{p}}{2^{q \alpha}-2^{p}}<\infty .
$$

Then in view of Forti's theorem, sequence $\left(H^{n}\left(F\left(G^{n}|x|\right)\right)\right)_{n \in \mathbb{N}}$ is a Cauchy sequence for every $x \in \mathcal{X}$ and thus so is $\left(2^{n p} F\left(2^{-n q}|x|\right)\right)_{n \in \mathbb{N}}$. Furthermore, the mapping (2.2) satisfies inequality (2.3). 
Next, we prove that $T$ solves (1.2). In fact, in (2.1) substitute $x$ with $2^{-n q} x$ also $y$ with $2^{-n q} y$, and fix arbitrarily $\tau, \eta \in[0, \infty)$. Then

$$
\begin{aligned}
& \| F\left(\frac{\left(\tau^{q}|x|\right) \Delta_{\mathcal{X}}^{*}\left(\eta^{q}|y|\right)}{2^{n q}}\right) \Delta_{\mathcal{Y}}^{*} F\left(\frac{\left(\tau^{q}|x|\right) \Delta_{\mathcal{X}}^{* *}\left(\eta^{q}|y|\right)}{2^{n q}}\right) \\
& -\left(\tau^{p} F\left(\frac{|x|}{2^{n q}}\right)\right) \Delta_{\mathcal{Y}}^{* *}\left(\eta^{p} F\left(\frac{|y|}{2^{n q}}\right)\right) \| \leq 2^{(p-1)} \vartheta\left(\left\|\frac{x}{2^{n q}}\right\|^{\alpha}+\left\|\frac{y}{2^{n q}}\right\|^{\alpha}\right) .
\end{aligned}
$$

Multiplying both sides of this last inequality by $2^{n p}$ yields

$$
\begin{aligned}
2^{n p} \| & F\left(\frac{\left(\tau^{q}|x|\right) \Delta_{\mathcal{X}}^{*}\left(\eta^{q}|y|\right)}{2^{n q}}\right) \Delta_{\mathcal{Y}}^{*} F\left(\frac{\left(\tau^{q}|x|\right) \Delta_{\mathcal{X}}^{* *}\left(\eta^{q}|y|\right)}{2^{n q}}\right) \\
& -\left(\tau^{p} F\left(\frac{|x|}{2^{n q}}\right)\right) \Delta_{\mathcal{Y}}^{* *}\left(\eta^{p} F\left(\frac{|y|}{2^{n q}}\right)\right) \| \leq \frac{\vartheta}{2^{(1-p)}} \frac{\|x\|^{\alpha}+\|y\|^{\alpha}}{2^{n(q \alpha-p)}} .
\end{aligned}
$$

Taking the limit in (3.2) we have via (2.2) that

$\left\|T\left(\left(\tau^{q}|x|\right) \Delta_{\mathcal{X}}^{*}\left(\eta^{q}|y|\right)\right) \Delta_{\mathcal{Y}}^{*} T\left(\left(\tau^{q}|x|\right) \Delta_{\mathcal{X}}^{* *}\left(\eta^{q}|y|\right)\right)-\left(\tau^{p} T(|x|)\right) \Delta_{\mathcal{Y}}^{* *}\left(\eta^{p} T(|y|)\right)\right\|=0$

for all $\tau, \eta \in[0, \infty)$ and all $x, y \in \mathcal{X}$, which is equivalent to (1.2). Thus $T$ also satisfies (1.3) in Remark 1.1. Finally we show the uniqueness, using a technique in [16]. In fact, assume that there is another functional $S: \mathcal{X} \rightarrow \mathcal{Y}$ which satisfies (1.2) and the inequality $\|S(|x|)-F(|x|)\| \leq \delta_{2}\|x\|^{\alpha_{2}}$ for some numbers $\alpha_{2}, \delta_{2} \in(0, \infty)$ with $q \alpha_{2}>p$, and for all $x \in \mathcal{X}$. In (2.3) let $\delta_{1}:=\frac{2^{p} \vartheta}{2^{q \alpha}-2^{p}}, \alpha_{1}:=\alpha$ and by choosing $\tau=2^{-n}$ in Remark 1.1 one can observe that for all $x \in \mathcal{X}$

$$
\begin{aligned}
\|S(|x|)-T(|x|)\|= & 2^{n p}\left\|S\left(2^{-n q}|x|\right)-T\left(2^{-n q}|x|\right)\right\| \\
\leq & 2^{n p}\left\|F\left(2^{-n q}|x|\right)-T\left(2^{-n q}|x|\right)\right\| \\
& +2^{n p}\left\|S\left(2^{-n q}|x|\right)-F\left(2^{-n q}|x|\right)\right\| \\
\leq & 2^{n p} \delta_{1}\left\|2^{-n q} x\right\|^{\alpha_{1}}+2^{n p} \delta_{2}\left\|2^{-n q} x\right\|^{\alpha_{2}} \\
= & 2^{\left(p-q \alpha_{1}\right) n} \delta_{1}\|x\|^{\alpha_{1}}+2^{\left(p-q \alpha_{2}\right) n} \delta_{2}\|x\|^{\alpha_{2}} .
\end{aligned}
$$

Hence

$$
\|S(|x|)-T(|x|)\| \leq 2^{\left(p-q \alpha_{1}\right) n} \delta_{1}\|x\|^{\alpha_{1}}+2^{\left(p-q \alpha_{2}\right) n} \delta_{2}\|x\|^{\alpha_{2}}
$$

which, in the limit, yields $\|S(|x|)-T(|x|)\|=0$ or equivalently $S(|x|)=T(|x|)$ for all $x \in \mathcal{X}$. 
This was to be proven.

Proof of Theorem 2.2. First, if we choose $\tau=\eta=2^{-1}, y=x$ and replace $x$ by $2^{q} x$ in inequality (2.4) then we obviously have

$$
\left\|2^{-p} F\left(2^{q}|x|\right)-F(|x|)\right\| \leq \beta+\vartheta 2^{q \alpha-p}\|x\|^{\alpha} .
$$

Next, let us define the following functions:

$$
\begin{array}{lll}
G: \mathcal{X}^{+} \rightarrow \mathcal{X}^{+}, & G(|x|)=2^{q}|x|, & \text { for all } x \in \mathcal{X}, \\
\delta: \mathcal{X}^{+} \rightarrow[0, \infty), & \delta(|x|)=\beta+\vartheta 2^{q \alpha-p}\|x\|^{\alpha}, & \text { for all } x \in \mathcal{X}, \\
\varphi:[0, \infty) \rightarrow[0, \infty), & \varphi(t)=2^{-p} t, & \\
H: \mathcal{Y}^{+} \rightarrow \mathcal{Y}^{+}, & H(|y|)=2^{-p}|y|, & \text { for all } y \in \mathcal{Y}, \\
d(\cdot, \cdot): \mathcal{Y}^{+} \times \mathcal{Y}^{+} \rightarrow[0, \infty), & d\left(\left|y_{1}\right|,\left|y_{2}\right|\right)=\left\|\left|y_{1}\right|-\left|y_{2}\right|\right\|, & \text { for all } y_{1}, y_{2} \in \mathcal{Y} .
\end{array}
$$

We shall verify the fulfilment of all the three conditions of the Forti's theorem as follows.

(I) From inequality (3.3) we obviously have

$$
\begin{aligned}
d(H(F(G(|x|))), F(|x|)) & =\|H(F(G(|x|)))-F(|x|)\| \\
& =\left\|2^{-p} F\left(2^{q}|x|\right)-F(|x|)\right\| \\
& \leq \beta+\vartheta 2^{q \alpha-p}\|x\|^{\alpha}=\delta(|x|) .
\end{aligned}
$$

(II) $d\left(H\left(\left|y_{1}\right|\right), H\left(\left|y_{2}\right|\right)\right)=2^{-p} \|\left|y_{1}\right|-\left|y_{2}\right|||=\varphi\left(d\left(\left|y_{1}\right|,\left|y_{2}\right|\right)\right)$ for all $y_{1}, y_{2} \in \mathcal{Y}$.

(III) Clearly, on the one hand $\varphi$ is a non-decreasing subadditive function on the positive half line, and on the other hand by applying Remark 2.2 on both the iterations $G^{j}$ and $\varphi^{j}$ of $G$ and $\varphi$ respectively, one can observe that

$$
\begin{aligned}
\sum_{j=0}^{\infty} \varphi^{j}\left(\delta\left(G^{j}(|x|)\right)\right) & =\beta \sum_{j=0}^{\infty} 2^{-p j}+\vartheta 2^{q \alpha-p}\|x\|^{\alpha} \sum_{j=0}^{\infty} 2^{(q \alpha-p) j} \\
& =\frac{\beta 2^{p}}{2^{p}-1}+\frac{\vartheta\|x\|^{\alpha} 2^{q \alpha}}{2^{p}-2^{q \alpha}}<\infty
\end{aligned}
$$

Then in view of Forti's theorem, sequence $\left(H^{n}\left(F\left(G^{n}|x|\right)\right)\right)_{n \in \mathbb{N}}$ is a Cauchy sequence for every $x \in \mathcal{X}$ and thus so is $\left(2^{-n p} F\left(2^{n q}|x|\right)\right)_{n \in \mathbb{N}}$. Furthermore, the mapping (2.5) satisfies inequality (2.6). 
Next, we prove that $T$ solves (1.2). In fact, in (2.4) substitute $x$ with $2^{n q} x$ also $y$ with $2^{n q} y$, and fix arbitrarily $\tau, \eta \in[0, \infty)$. Then

$$
\begin{aligned}
& \| F\left(2^{n q}\left(\left(\tau^{q}|x|\right) \Delta_{\mathcal{X}}^{*}\left(\eta^{q}|y|\right)\right)\right) \Delta_{\mathcal{Y}}^{*} F\left(2^{n q}\left(\left(\tau^{q}|x|\right) \Delta_{\mathcal{X}}^{* *}\left(\eta^{q}|y|\right)\right)\right) \\
&-\left(\tau^{p} F\left(2^{n q}|x|\right)\right) \Delta_{\mathcal{Y}}^{* *}\left(\eta^{p} F\left(2^{n q}|y|\right)\right) \| \\
& \leq \beta+2^{-(p+1)} \vartheta\left(\left\|2^{n q} x\right\|^{\alpha}+\left\|2^{n q} y\right\|^{\alpha}\right) .
\end{aligned}
$$

Dividing both sides of this last inequality by $2^{n p}$ yields

$$
\begin{gathered}
\| \frac{F\left(2^{n q}\left(\left(\tau^{q}|x|\right) \Delta_{\mathcal{X}}^{*}\left(\eta^{q}|y|\right)\right)\right) \Delta_{\mathcal{Y}}^{*} F\left(2^{n q}\left(\left(\tau^{q}|x|\right) \Delta_{\mathcal{X}}^{* *}\left(\eta^{q}|y|\right)\right)\right)}{2^{n p}} \\
-\frac{\left(\tau^{p} F\left(2^{n q}|x|\right)\right) \Delta_{\mathcal{Y}}^{* *}\left(\eta^{p} F\left(2^{n q}|y|\right)\right)}{2^{n p}} \| \\
\leq \beta 2^{-n p}+2^{-(p+1)} \vartheta\left(\|x\|^{\alpha}+\|y\|^{\alpha}\right) 2^{(q \alpha-p) n} .
\end{gathered}
$$

Taking the limit in (3.4) we have via (2.5) that

$$
\left\|T\left(\left(\tau^{q}|x|\right) \Delta_{\mathcal{X}}^{*}\left(\eta^{q}|y|\right)\right) \Delta_{\mathcal{Y}}^{*} T\left(\left(\tau^{q}|x|\right) \Delta_{\mathcal{X}}^{* *}\left(\eta^{q}|y|\right)\right)-\left(\tau^{p} T(|x|)\right) \Delta_{\mathcal{Y}}^{* *}\left(\eta^{p} T(|y|)\right)\right\|=0
$$

for all $\tau, \eta \in[0, \infty)$ and all $x, y \in \mathcal{X}$, which is equivalent to (1.2). Thus $T$ satisfies (1.3) in Remark 1.1. Finally we show the uniqueness, using a technique in [16]. In fact, assume that there is another functional $S: \mathcal{X} \rightarrow \mathcal{Y}$ which satisfies (1.2) and the inequality $\|S(|x|)-F(|x|)\| \leq \beta_{2}+\delta_{2}\|x\|^{\alpha_{2}}$ for some numbers $\alpha_{2}, \delta_{2} \in(0, \infty), \beta_{2} \in[0, \infty)$ with $q \alpha_{2}<p$, and for all $x \in \mathcal{X}$. In (2.6) let $\beta_{1}:=\frac{\beta 2^{p}}{2^{p}-1}, \delta_{1}:=\frac{\vartheta 2^{q \alpha}}{2^{p}-2^{q \alpha}}, \alpha_{1}:=\alpha$ and by choosing $\tau=2^{n}$ in Remark 1.1 one can observe that for all $x \in \mathcal{X}$

$$
\begin{aligned}
\|S(|x|)-T(|x|)\|= & 2^{-n p}\left\|S\left(2^{n q}|x|\right)-T\left(2^{n q}|x|\right)\right\| \\
\leq & 2^{-n p}\left\|F\left(2^{n q}|x|\right)-T\left(2^{n q}|x|\right)\right\| \\
& +2^{-n p}\left\|S\left(2^{n q}|x|\right)-F\left(2^{n q}|x|\right)\right\| \\
\leq & 2^{-n p}\left(\beta_{1}+\delta_{1}\left\|2^{n q} x\right\|^{\alpha_{1}}\right)+2^{-n p}\left(\beta_{2}+\delta_{2}\left\|2^{n q} x\right\|^{\alpha_{2}}\right) \\
= & 2^{-n p}\left(\beta_{1}+\beta_{2}\right)+\delta_{1} 2^{\left(q \alpha_{1}-p\right) n}\|x\|^{\alpha_{1}}+\delta_{2} 2^{\left(q \alpha_{2}-p\right) n}\|x\|^{\alpha_{2}} .
\end{aligned}
$$

Hence

$$
\|S(|x|)-T(|x|)\| \leq 2^{-n p}\left(\beta_{1}+\beta_{2}\right)+\delta_{1} 2^{\left(q \alpha_{1}-p\right) n}\|x\|^{\alpha_{1}}+\delta_{2} 2^{\left(q \alpha_{2}-p\right) n}\|x\|^{\alpha_{2}}
$$


which, in the limit, yields $\|S(|x|)-T(|x|)\|=0$ or equivalently $S(|x|)=T(|x|)$ for all $x \in \mathcal{X}$. This completes the proof.

To end our paper we give an example showing that stability fails to occur in general.

ExAmPle 1. Fix arbitrarily $\tau, \eta \in(0,2)$ and consider the function

$$
F:[0, \infty) \rightarrow[0, \infty), F(x)=x^{\alpha+1}, \quad \alpha=\frac{p}{q} .
$$

Since $F$ is increasing the first equality in the chain below is valid, entailing the subsequent relations:

$$
\begin{aligned}
\mid F\left(\left(\tau^{q} x\right) \vee\left(\eta^{q} y\right)\right) & -\left(\tau^{p} F(x)\right) \wedge\left(\eta^{p} F(y)\right) \mid \\
& =\left|\left(\tau^{q} x\right)^{\alpha+1} \vee\left(\eta^{q} y\right)^{\alpha+1}-\left(\tau^{p} x^{\alpha+1}\right) \wedge\left(\eta^{p} y^{\alpha+1}\right)\right| \\
& \leq\left(\tau^{q} x\right)^{\alpha+1} \vee\left(\eta^{q} y\right)^{\alpha+1}+\left(\tau^{p} x^{\alpha+1}\right) \wedge\left(\eta^{p} y^{\alpha+1}\right) \\
& \leq\left(2^{q} x\right)^{\alpha+1} \vee\left(2^{q} y\right)^{\alpha+1}+\left(2^{p} x^{\alpha+1}\right) \wedge\left(2^{p} y^{\alpha+1}\right) \\
& \leq 2^{p+q}\left(x^{\alpha+1} \vee y^{\alpha+1}\right)+2^{p+q}\left(x^{\alpha+1} \wedge y^{\alpha+1}\right) \\
& =2^{p+q}\left(x^{\alpha+1}+y^{\alpha+1}\right)
\end{aligned}
$$

for all $x, y \in[0, \infty)$. Now, let $T:[0, \infty) \rightarrow[0, \infty)$ be a function such that $T\left(\mu^{q} x\right)=\mu^{p} T(x)$ for all $x \in[0, \infty)$ and all $\mu \in[0, \infty)$. Since $x=\left(x^{1 / q}\right)^{q}$, and $\alpha$ is the ratio of $p$ and $q$, we can then note that $T(x)=x^{\alpha} T(1)$ for every $x \in[0, \infty)$. Now,

$$
\begin{aligned}
\sup _{x \in(0, \infty)} \frac{|F(x)-T(x)|}{2^{p+q} x^{\alpha+1}} & =\sup _{x \in(0, \infty)} \frac{\left|x^{\alpha+1}-T\left(\left(x^{\frac{1}{q}}\right)^{q}\right)\right|}{2^{p+q} x^{\alpha+1}} \\
& =\sup _{x \in(0, \infty)} \frac{\left|x^{\alpha+1}-x^{\alpha} T(1)\right|}{2^{p+q} x^{\alpha+1}} \\
& =\frac{1}{2^{p+q}} \sup _{x \in(0, \infty)}\left|1-\frac{T(1)}{x}\right|=\infty .
\end{aligned}
$$

The above example about the lack of stability on the real line in lattice environments is the counterpart of the example given by S. Czerwik [9] in the addition environments for quadratic mappings. 


\section{ACKNOWLEDGEMENTS}

The second author is supported by the Hungarian Academy of Sciences, and OTKA Grant PD124875.

\section{REFERENCES}

[1] N.K. Agbeko, On optimal averages, Acta Math. Hungar. 63 (2) (1994), 133 147.

[2] N.K. Agbeko, On the structure of optimal measures and some of its applications, Publ. Math. Debrecen 46 (1-2) (1995), 79-87.

[3] N.K. AgBeko, Stability of maximum preserving functional equations on Banach lattices, Miskolc Math. Notes 13 (2) (2012), 187-196.

[4] N.K. Agbeko, S.S. Dragomir, The extension of some Orlicz space results to the theory of optimal measure, Math. Nachr. 286 (8-9) (2013), $760-771$.

[5] N.K. AgBeko, The Hyers-Ulam-Aoki type stability of some functional equation on Banach lattices, Bull. Pol. Acad. Sci. Math. 63 (2) (2015), 177-184.

[6] N.K. AgBeko, A remark on a result of Schwaiger, Indag. Math. (N.S.) 28 (2) (2017), 268-275.

[7] N.K. Agbeko, W. Fechner, E. Rak, On lattice-valued maps stemming from the notion of optimal average, Acta Math. Hungar. 152 (1) (2017), $72-$ 83.

[8] T. Aoki, Stability of the linear transformation in Banach spaces, J. Math. Soc. Japan 2 (1-2) (1950), 64-66.

[9] St. Czerwik, On the stability of the quadratic mapping in normed spaces, Abh. Math. Sem. Hamburg 62 (1992), 59-64.

[10] G.-L. ForTI, Comments on the core of the direct method for proving HyersUlam stability of functional equations, J. Math. Anal. Appl. 295 (1) (2004), $127-133$.

[11] Z. GAJDA, On stability of additive mappings, Internat. J. Math. Sci. 14(3) (1991), 431-434.

[12] P. Găvruţa, On a problem of G. Isac and Th. M. Rassias concerning the stability of mappings, J. Math. Anal. Appl. 261 (2) (2001), 543-553.

[13] D.H. Hyers, On the stability of the linear functional equation, Proc. Nat Acad. Sci. USA 27 (4) (1941), 222-224.

[14] M. LaczKOvich, The local stability of convexity, affinity and of the Jensen equation, Aequationes Math. 58(1-2) (1999), 135-142.

[15] Zs. PÁLES, Hyers-Ulam stability of the Cauchy functional equation on squaresymmetric groupoids, Publ. Math. Debrecen 58 (4) (2001), 651-666.

[16] N. SAlehi, S.M.S. ModarREs, Stablity of maximum preserving quadratic functional equation in Banach lattices, Miskolc Math. Notes 17 (1) (2016), $581-589$. 